\title{
Nivolumab: 5 Years Since FDA Approval of the First Checkpoint Inhibitor for Renal Cell Carcinoma
}

\author{
Ilya Tsimafeyeu* \\ Institute of Oncology Hadassah Moscow, Kidney Cancer Research Bureau, Moscow, Russia
}

Received 6 January 2021

Accepted 16 February 2021

Pre-press 4 March 2021

Published 16 June 2021

\begin{abstract}
On November 23, 2015, the US Food and Drug Administration (FDA) approved nivolumab for the treatment of metastatic renal cell carcinoma (RCC), thus opening a new era of immunotherapy for this tumor. This review summarizes the 5-year experience of studying and using nivolumab in RCC patients.
\end{abstract}

Keywords: Nivolumab, metastatic renal cell carcinoma

\section{INTRODUCTION}

Renal cell carcinoma (RCC), along with melanoma, has traditionally been regarded as a model for the study of new immunotherapy approaches. On the one hand, tumor-associated antigens activate adaptive immunity [1]. On the other hand, the system of regulatory mechanisms causes immunosuppression and deactivation of the developing antitumor response [2, 3]. The presence of immune checkpoints has previously been shown to be responsible for an aggressive phenotype of RCC. In particular, the expression of PD-1, PD-L1, and CTLA-4 receptors on cells of both primary tumor and metastases leads to poorer overall survival rates in patients with metastatic RCC [4-6]. At the ESMO congress 2020, new data were presented on the effect of the "immune

\footnotetext{
*Correspondence to: Ilya Tsimafeyeu, MD, Institute of Oncology Hadassah Moscow, Kidney Cancer Research Bureau, Bolshoi Boulevard, Building 46, Stroenie 1, Moscow, 143026, Russia; E-mail: i.tsimafeyeu@hadassah.moscow.
}

tumor phenotype" consisting of a high number of T cells and a small number of angiogenic and stromal factors on the activation of the immune system and the efficacy of its stimulation [7].

Back in the 2000s, it was assumed that the combination of two immunotherapy agents (back then - cytokines) would increase the overall efficacy of treatment [8]. Repeated attempts were made to combine interferon and interleukin-2 in different regimens in studies, but the results were often so contradictory that monotherapy with cytokines remained the standard of that time [9]. The second direction was the study of combinations of cytokines, colonystimulating factors, with vaccines, which also did not deliver a significant result [10].

Five years ago, on November 23, 2015, the US Food and Drug Administration (FDA) approved nivolumab, a monoclonal antibody that blocks the PD-1 receptor, for the treatment of metastatic RCC, thus opening a new era of immunotherapy for this tumor [11]. Immunotherapy has taken a new turn, 
Table 1

Nivolumab in second- and later-line therapy

\begin{tabular}{|c|c|c|c|}
\hline Results / Study & $\begin{array}{c}\text { CheckMate } 010 \\
\qquad(\mathrm{~N}=54) \\
\text { Phase } 2 \text {, } \\
\text { Third-line therapy, } \\
\text { Nivolumab } 2 \mathrm{mg} / \mathrm{kg}\end{array}$ & $\begin{array}{c}\text { CheckMate } 025 \\
(\mathrm{~N}=410) \\
\text { Phase } 3 \text {, } \\
\text { Second- and third- } \\
\text { line therapy, } \\
\text { Nivolumab } 3 \mathrm{mg} / \mathrm{kg}\end{array}$ & $\begin{array}{c}\text { NIVOREN GETUG-AFU } 26 \\
(\mathrm{~N}=720) \\
\text { Phase } 2, \text { Two and } \\
\text { more previous lines, } \\
\text { Real-world } \\
\text { population, } \\
\text { Nivolumab } 3 \mathrm{mg} / \mathrm{kg}\end{array}$ \\
\hline Overall survival, median, months & 25.5 & 25.8 & 24.5 \\
\hline Progression-free survival, median, months & 4.0 & 4.2 & 3.2 \\
\hline Objective response rate, $\%$ & 22 & 23 & 21 \\
\hline Grade $3-4$ adverse events, $\%$ & 17 & 21.4 & 17.9 \\
\hline
\end{tabular}

with new hopes for combining multiple checkpoint inhibitors with targeted agents. Nivolumab and ipilimumab, pembrolizumab or avelumab in combination with axitinib, and nivolumab with cabozantinib have changed the practical guidelines for metastatic RCC.

This review summarizes the 5-year experience of studying and using nivolumab in RCC patients.

\section{NIVOLUMAB IN SECOND- AND LATER-LINE THERAPY}

The efficacy and toxicity studies of nivolumab in metastatic RCC were started in patients who experienced disease progression on targeted therapy in previous lines (Table 1).

In a Phase 2 study, 168 patients with metastatic RCC previously treated with VEGFR inhibitors were randomized in a $1: 1: 1$ ratio to receive nivolumab $0.3 \mathrm{mg} / \mathrm{kg}(n=60), 2 \mathrm{mg} / \mathrm{kg}(n=54), 10 \mathrm{mg} / \mathrm{kg}(n=$ 54) [12]. The compound was administered intravenously once a week every 3 weeks. The primary efficacy endpoint was progression-free survival, secondary endpoints were objective response rate, overall survival, and safety. The median progression-free survival was 2.7 months, 4.0 months, 4.2 months for three dose levels, respectively $(\mathrm{P}=0.9)$. Objective response rates were $20 \%, 22 \%$ and $20 \%$ in groups, the median overall survival was 18.2 months, 25.5 months, 24.7 months, respectively. The most common side effect of therapy was fatigue (24\%, $22 \%, 35 \%$ ). Grade 3-4 toxicity was observed only in $18(11 \%)$ patients. The authors concluded that nivolumab was well tolerated and had antitumor efficacy regardless of the dose escalation.

In 2015, the first results of a randomized phase 3 trial of nivolumab (CheckMate 025) were published [13]. This study enrolled 821 patients with metastatic RCC and disease progression on the first- or second- line therapy with antiangiogenic agents. Patients were randomized into 2 groups: one to receive nivolumab ( $3 \mathrm{mg} / \mathrm{kg}$, intravenously, every 2 weeks), and the other one to receive everolimus (10 $\mathrm{mg}$ orally). Overall survival was the primary efficacy endpoint. In addition, the authors evaluated the objective response rate, progression-free survival and safety of the antibody. At 2020 GU ASCO, the authors presented the final results of the CheckMate 025 study. For the first time, a long-awaited 5-year overall survival rate was analyzed in patients with metastatic RCC treated with the checkpoint inhibitor, which amounted to $26 \%$, with its median being 25.8 months [14]. These results were definitely better than historical data before the era of immunotherapy. For example, the 5-year overall survival rate was $8.2 \%$ in the RENSUR5 register [15] and $12 \%$ in the SEER database [16]. It should be noted that both RENSUR5 and SEER analyzed patients with newly diagnosed advanced RCC, while CheckMate 025 included patients with disease progression on standard therapy; however, its result was better even in these settings.

The objective response rate was also satisfactory, amounting to $23 \%$. If patients achieved response to treatment, the median response duration was 18.2 months. The median progression-free survival was 4.2 months.

Grade 3-4 adverse events were reported in 19\% of patients treated with nivolumab [13]. Over 5 years, the incidence of these adverse events increased to only $21 \%$, which theoretically indicates the absence of long-term toxicity. Out of all grade 3-4 adverse events, patients in the nivolumab group most often experienced fatigue (2\%). Other adverse events in patients treated with nivolumab included cough, nausea, rash, dyspnea, diarrhea, constipation, decreased appetite, back pain and joint pain. No drastic changes in various types of toxicity were observed either over time. 
Therefore, nivolumab monotherapy was a 5 times more effective therapeutic option with lower toxicity than targeted agents. Moreover, nivolumab has been associated with improved patient quality of life compared with everolimus [17]. In terms of FKSI-DRS score, more patients had a clinically meaningful health-related quality of life improvement with nivolumab (55\%) versus everolimus (37\%; $P<0.0001$ ).

The efficacy and toxicity of nivolumab in subsequent lines of therapy have also been confirmed in a prospective phase 2 study NIVOREN GETUG-AFU 26 [18]. It enrolled patients with characteristics that were as close to real world setting ones as possible. For example, $22.4 \%$ of patients had received more than 2 previous lines of therapy, $21.3 \%$ of patients had received mTOR inhibitors in previous lines, $15 \%$ of patients had ECOG PS 2, 12.3\% of patients had asymptomatic brain metastases, $34.3 \%$ of patients had renal dysfunction and finally $25.5 \%$ of patients were from the poor prognosis group according to the IMDC criteria. With a median follow-up of 20.9 months and analysis of data from 720 patients, the incidence of treatment-related adverse events was $17.1 \%$, which was lower than in the pivotal CheckMate 025 study (20\%) [13]. Complications leading to discontinuation of therapy were observed only in $7.5 \%$ of patients. The objective response rate was $20.8 \%$. The median progression-free survival was 3.2 months. The one-year overall survival rate was $69 \%$. The median overall survival was 32.8 months, 25.0 months and 10.4 months in patients with favorable risk, intermediate risk and in the poor prognosis group, respectively. The authors concluded that the safety and efficacy of nivolumab in "real world setting" is comparable to the results of the phase 3 study. In patients with ECOG PS 2, the overall survival was lower $(P<0.0001)$ [19]. The number of prior treatment lines had no effect on progression-free survival or overall survival. The use everolimus in prior lines had a negative impact on survival $(P=0.04)$. The efficacy of the drug did not depend on the creatinine clearance $(<60 \geq)$ and the presence of brain metastases. For example, the response rate for intracranial metastases was $12 \%$ [20]. The median time to disease control ranged from 2.7 to 4.8 months, and the 12 -month overall survival was $59 \%$ to $67 \%$, depending on the previous treatment. Nivolumab was well tolerated in this group without unexpected toxicity.

The efficacy and safety of nivolumab in patients with chronic hepatitis $\mathrm{C}$ virus, who are usually excluded from clinical trials, was evaluated in a cohort study [21]. A total of 44 eligible patients were enrolled. The groups of patients with hepatitis $(\mathrm{N}=22$, study cohort) and without hepatitis $(\mathrm{N}=22$, control cohort) were well balanced. The overall survival and progression-free survival in patients infected with hepatitis $\mathrm{C}$ were at least non-inferior to those in patients without hepatitis. Thus, the median overall survival was 27.5 and 21.7 months in the test and control groups, respectively $(P=0.005$ in favor of the test group). The median progression-free survival was 7.5 and 4.9 months $(P=0.013$ in favor of the test group). Despite the absence of differences in the objective response rates between the groups ( $27 \%$ vs. $23 \%, P=0.7$ ), patients with hepatitis had significantly more sustained responses $(P=0.01)$. Nivolumab was well tolerated by all HCV-positive patients. No unexpected toxicity was observed. The viral load assessment during nivolumab therapy was available in 14 of $22(64 \%)$ patients with hepatitis C. Nivolumab did not significantly affect hepatitis virus concentrations (mean change of $210 \mathrm{IU} / \mathrm{mL}$, $P=0.82)$ in the absence of antiviral therapy.

These findings served as the basis for the development of a nivolumab combination for the use in the first-line therapy.

\section{NIVOLUMAB PLUS IPILIMUMAB AS FIRST-LINE TREATMENT}

After the success of immunotherapy in patients with disease progression on conventional targeted therapy, it was logical to study the efficacy of the novel method of first-line therapy. Moreover, the use of a combination of inhibitors blocking two checkpoints, PD-1 and CTLA-4, seemed appropriate. Since the first line of therapy makes the maximum contribution to the overall survival of patients with metastatic RCC, and knowing that there is a significant effect on the survival in the second-line, it could be assumed that nivolumab in combination with ipilimumab would significantly improve the overall outcome of treatment of newly diagnosed metastatic RCC. Finally, let us suppose that if a patient develops metastases, this means that the tumor cells have escaped immune surveillance and, therefore, an immediate effect on the immune system in the first-line of therapy is needed.

In the randomized phase 3 pivotal CheckMate 214 study [22], treatment-naive patients with metastatic clear-cell RCC were randomized to receive nivolumab plus ipilimumab $(\mathrm{N}=550)$ or sunitinib 
$(\mathrm{N}=546)$. The study was designed to evaluate the efficacy of therapy in the intermediate and poor prognosis groups according to IMDC criteria. Nivolumab was used at a dose of $3 \mathrm{mg} / \mathrm{kg}$ in combination with ipilimumab at a dose of $1 \mathrm{mg} / \mathrm{kg}$ every 3 weeks, a total of 4 doses, followed by therapy with nivolumab at a dose of $3 \mathrm{mg} / \mathrm{kg}$ every 2 weeks. Patients received sunitinib $50 \mathrm{mg}$ on a standard $4 / 2$ schedule. The primary endpoints in the study were overall survival, progression-free survival, and objective response rates in the group of patients with intermediate and poor risk.

The median overall survival in the sunitinib group was announced back in 2018 and amounted 26.6 months. It is surprising and at the same time significant that the median survival in the combination therapy group was not achieved over two yearsmore than $50 \%$ of patients remained alive. The authors presented the results of the study with a minimum follow-up of 48 months at the 2020 ESMO Congress [23]. The median overall survival in the nivolumab-ipilimumab group was 48.1 months. Investigators found statistically significant benefits in favor of nivolumab plus ipilimumab over sunitinib, with a $35 \%$ reduction in mortality risk $(\mathrm{HR}=$ $0.65 ; P<0.0001)$ in patients with intermediate and poor prognosis. The 4-year overall survival was $50 \%$ in the immunotherapy group and $35.8 \%$ in the sunitinib group. Objective response rates were also significantly higher for nivolumab plus ipilimumab: $41.9 \%$ versus $26.8 \%(P<0.0001) .10 .4 \%$ of patients achieved complete tumor regression during immunotherapy. Treatment responses, both partial and complete, were $55 \%$ longer with nivolumab and ipilimumab than with sunitinib. The median progression-free survival was 11.2 months in the nivolumab plus ipilimumab group. Interestingly, the $35 \%$ survival tail seen with nivolumab/ipilimumab treatment has not been seen with other combinations so far. This may indicate the prolonged effects of the two immune checkpoint inhibitors. In addition, the long-term tail for the monotherapy with nivolumab is well below $10 \%$, suggesting that two antibodies are required to optimize treatment outcome in RCC.

The data on the efficacy of the combination in patients without previous cytoreductive nephrectomy appear to be fascinating. The CheckMate 214 study included 108 patients with target kidney lesions who, for some reason, were not candidates for cytoreductive nephrectomy. Such patients are known to have a worse prognosis in terms of survival, which is also confirmed by the characteristics of the patients included in the analysis: the majority were from the IMDC intermediate and poor prognosis groups (only $2 \%$ of patients had a favorable prognosis), and, therefore, it was especially important to evaluate the efficacy of double immunotherapy combination in this subgroup of patients. At the time of data evaluation (median follow-up of 48 months), $35 \%$ of patients on nivolumab plus ipilimumab experienced a tumor size reduction of more than $30 \%$ (vs. $20 \%$ in the sunitinib group), the objective response rate was $34 \%$ and $14.5 \%$ in these groups, respectively, and the same proportions of patients $(34 \%$ and $14.5 \%)$ achieved partial response; there were no complete responses in any of the groups. The median duration of response to immunotherapy was 20.5 months vs. 14.1 months on sunitinib $(\mathrm{HR}=0.69)$, and median overall survival was 26.1 months vs. 14.3 months, respectively $(\mathrm{HR}=0.63)$. Thus, patients with target kidney lesions responded better to combination therapy than to sunitinib therapy: the objective response rate was higher, the responses were deeper and more prolonged.

Could we have previously expected metastatic RCC patients with poor and intermediate prognosis to live for 4 years? Undoubtedly, this figure appears to be more attractive than previously demonstrated ones in population retrospective analyses. For example, in the Russian registry study RENSUR3, which involved 573 patients in approximately the same period (20152016) as in the CheckMate 214 study (2014-2016), the 3 -year overall survival rate was only $21 \%$, and the median was 12 months [24].

The results obtained for therapy with nivolumab plus ipilimumab in patients with sarcomatoid renal cell carcinoma were even more impressive. These patients $(n=145)$ were also treated in the CheckMate 214 study [25]. It should be noted that, like patients without previous cytoreductive nephrectomy, patients with sarcomatoid RCC represent a serious medical and social issue, as they have a significantly worse prognosis for survival and limited treatment options. In the current analysis, the vast majority of patients $(n=139)$ were from the intermediate and poor prognosis group, which was undoubtedly attributable to the histological characteristics of the tumor. Currently, data from a 42-month follow-up period for this cohort of patients are presented. The median overall survival in patients treated with nivolumab plus ipilimumab was not achieved, while being 14.2 months ( $\mathrm{HR}=0.45$, $p=0.0004)$ in patients treated with sunitinib. The median progression-free survival on nivolumab plus 
Table 2

Nivolumab plus ipilimumab as first-line treatment

\begin{tabular}{lccc}
\hline Results / Study & $\begin{array}{c}\text { CheckMate 241 } \\
(\mathrm{N}=425) \\
\text { Intermediate and } \\
\text { poor risk group }\end{array}$ & $\begin{array}{c}\text { CheckMate 241 } \\
(\mathrm{N}=550) \\
\text { ITT population }\end{array}$ & $\begin{array}{c}\text { CheckMate 241 } \\
(\mathrm{N}=54) \\
\text { Sarcomatoid } \\
\text { features }\end{array}$ \\
\hline Overall survival, median, months & 48.1 & $\mathrm{NR}$ & $\mathrm{NR}$ \\
Progression-free survival, median, months & 11.2 & 12.2 & 26.5 \\
Objective response rate, \% & 41.9 & 39.1 & 60.8 \\
Grade 3-4 adverse events, \% & - & 47.9 & - \\
\hline
\end{tabular}

ipilimumab therapy is more than 5 times higher than that on sunitinib therapy-26.5 months vs. 5.1 months, respectively $(\mathrm{HR}=0.54, p=0.0093)$. The objective response rate in patients on combination therapy reaches $60.8 \%$ vs. $23.1 \%$ in the sunitinib group, with complete response rates of $18.9 \%$ vs. $3.1 \%$, respectively. Thus, nivolumab and ipilimumab shows unprecedented long-term survival and objective response rates, including complete responses, which allows recommending this therapeutic option as preferable for treatment-naive patients with sarcomatoid renal cell carcinoma with intermediate and poor prognosis.

Efficacy analysis was conducted in intention-totreat patients and are presented in favorable risk group [26]. Superior overall survival with nivolumab and ipilimumab was sustained in the intention-to-treat population $(\mathrm{HR}=0.69)$. In patients with favorable risk, the difference in overall survival was not statistically significant $(H R=0.93)$ and survival probabilities at 4 years were similar.

With regard to the toxicity of the combination, it was acceptable in all studied subgroups. The longterm follow-up showed the incidence of grade 3-4 adverse events in the nivolumab + ipilimumab and sunitinib groups of $47.9 \%$ and $64.1 \%$, respectively. This trend is in line with the 2020 American Society of Clinical Oncology (ASCO) report that the new combinations show superior efficacy in reducing toxicity than previous treatments. Remarkably, even when patients discontinued immunotherapy due to toxicity (22\%), the overall survival did not decrease [26]. The most common grade 3-4 adverse events associated with immunotherapy were increased lipase $(10 \%)$, amylase $(6 \%)$, and alanine aminotransferase levels $(5 \%)$, while in the sunitinib group, these were hypertension $(17 \%)$, fatigue $(10 \%)$ and palmar-plantar erythrodysesthesia (9\%) [22]. The incidence of all grade 3-4 adverse events was $47 \%$ and $64 \%$ in the immunotherapy and sunitinib groups, respectively. High-dose glucocorticoids were required in $35 \%$ of patients. Thus, based on the results of the CheckMate
214 study, the combination of nivolumab and ipilimumab was approved in Russia and other countries for use in patients with metastatic clear cell RCC from the intermediate and poor prognosis group. The combination was also included in Russian and international guidelines [27, 28]. Table 2 summarizes the results of the CheckMate 214 study.

Is ipilimumab needed? The authors of the phase 2 OMNIVORE study suggested that not all patients might need a CTLA-4 inhibitor [29]. In addition, the optimal duration of maintenance therapy with nivolumab in responders is also unknown. Using an adaptive study design, they evaluated the efficacy of sequential addition of two doses of ipilimumab in patients who did not immediately respond to nivolumab alone and the duration of nivolumab treatment in responders. All patients $(\mathrm{N}=83)$ received nivolumab at the first stage for 6 months, followed by a response assessment and decision making. Most of the patients had a favorable ECOG status, clear-cell RCC, IMDC intermediate/poor prognosis. Half of the patients had not previously received systemic therapy. At 6 months, induction therapy with nivolumab resulted in confirmed partial response in $14 \%$ of patients $(12 / 83 ; 17 \%(7 / 42)$ in untreated patients, $12 \%(5 / 41)$ in treated patients). In these patients, nivolumab therapy was interrupted and $45 \%$ did not resume it for a year as the response persisted. Out of $57(69 \%)$ patients who received addition of 2 doses of ipilimumab, two who had previously experienced disease progression on nivolumab monotherapy developed partial response $(4 \%)$. However, $40 \%$ of patients experienced disease progression with the addition of ipilimumab. The 18-month overall survival rate was $79 \%$. Treatment-related grade 3-4 adverse events occurred in $7 \%$ of cases with nivolumab induction and in $23 \%$ with subsequent addition of ipilimumab. The investigators believe that it is currently premature to recommend the described strategy in routine practice. Delayed addition of ipilimumab led to response in only $4 \%$ of patients, and there were no complete responses in the study, the 
development of which is well known with the simultaneous use of the combination of nivolumab plus ipilimumab. Despite patients could achieve sustained response after induction with nivolumab, therapy had to be resumed in half of the cases.

A very similar phase 2 HCRN GU16-260 study resulted in more optimistic conclusions [30]. One hundred twenty-three patients with clear-cell RCC from all prognostic groups (favorable prognosis $-25 \%$, intermediate prognosis $-65 \%$, poor prognosis $-10 \%)$ received the first-line monotherapy with nivolumab $240 \mathrm{mg}$ every 2 weeks (6 doses), then $360 \mathrm{mg}$ every 3 weeks ( 4 doses) followed by $480 \mathrm{mg}$ every 4 weeks until disease progression or unacceptable toxicity. The objective response rate was $31.7 \%$, including complete response in 5.7\% $(13.3 \%$ in the favorable prognosis group). The median duration of response was 19.3 months, and the median progression-free survival was 8.3 months. The median progression-free survival increased to 19.3 months in patients with favorable prognosis. 65 patients with disease progression or stable disease on nivolumab monotherapy were to be treated with ipilimumab ( $1 \mathrm{mg} / \mathrm{kg}$ every 3 weeks, 4 doses $)$ followed by maintenance therapy with nivolumab $(3 \mathrm{mg} / \mathrm{kg}$ every 2 weeks). However, 31 patients could not be switched on this therapy due to immune-mediated adverse events, symptomatic disease progression or initiation of another treatment. The addition of ipilimumab resulted in the development of response (partial in all cases) in $13.3 \%$ patients and stable disease in $23.3 \%$ patients, with the incidence of grade $\geq 3$ adverse events being $40 \%$. $81 \%$ of patients were alive at the time of the last assessment. The investigators concluded that nivolumab monotherapy can be used in some patients, such as those with potential intolerance to first-line ipilimumab or tyrosine kinase inhibitors, and in patients with favorable prognosis. However, the combination of nivolumab and ipilimumab remains the most preferred because of the high objective response rates, including longer complete responses and longer disease control times.

Finally, European multicenter study (TITAN) enrolled 258 patients with IMDC intermediate and poor risk, advanced clear-cell RCC, and disease progression after no more two previous treatment lines [31]. Patients started with nivolumab induction. In case of early progression of disease (week 8) or either stable disease or progression at week 16, patients received nivolumab and ipilimumab boost cycles. Responders to nivolumab monotherapy continued with maintenance with nivolumab and ipilimumab boosts only for progression. In first-line therapy, a tailored approach using combination of two antibodies significantly improved objective response rate compared to nivolumab alone.

How effective is the combination of nivolumab plus ipilimumab in patients with progression on prior treatment with checkpoint inhibitors? The answer to this question was obtained in the phase 2 FRACTION-RCC study, in which 46 patients with progression on CTLA-4, PD-1, PD-L1, LAG3 inhibitors received nivolumab plus ipilimumab in the standard regimen [32]. Also, $80 \%$ of patients had previously received tyrosine kinase inhibitors. The objective response rate, being the primary endpoint, was $15.2 \%$, and disease control was achieved in $52.2 \%$ of patients. The median progression-free survival was 16.1 months. $28 \%$ of patients reported grade 3-4 adverse events, with diarrhea being most common (9\%). Only three patients had to interrupt therapy due to toxicity. Therefore, although the efficacy of nivolumab and ipilimumab in patients with disease progression on checkpoint inhibitors was not as successful as that seen in patients treated in the CheckMate 214 study, these results help fill the data gap regarding sequential therapy. Overall, the FRACTION-RCC study offers an effective new strategy for evaluating cancer immunotherapies in heavily pretreated patients with metastatic RCC.

Another interesting approach is the use of the combination of nivolumab plus ipilimumab for an immunogenic tumor phenotype.

Perhaps, therapy will be selected on the basis of a molecular tumor subtype in the near future. In a randomized phase 2 BIONIKK study, treatment with nivolumab, ipilimumab, or a tyrosine kinase inhibitor was prescribed on the basis of "immune" or "angiogenic" phenotypes determined using a 35-gene expression signature and responsible for respective strong or weak characteristics [33]. 202 patients were randomized to receive nivolumab, nivolumab plus ipilimumab, or targeted therapy. The primary endpoint was the objective response rate in each group, which ranged from $21 \%$ to $54 \%$ depending on the phenotype. The authors showed that gene expression signatures can increase response rates. They are planning an extensive translation program to identify new biomarkers.

Also, stereotactic radiotherapy as a complement to immunotherapy should not be dismissed. Irradiation of even one or more lesions can help activate the immune system by releasing antigens, thereby increasing the efficacy of checkpoint inhibitors [34, 
35]. In the RADVAX study, 25 patients with clear cell metastatic RCC received standard doses of nivolumab and ipilimumab, followed by nivolumab monotherapy [36]. Stereotactic radiotherapy was given on 1-2 metastases at a total dose of $50 \mathrm{~Gy}$ in 5 fractions between the first and second doses of nivolumab and ipilimumab. The primary objectives of this study were to determine the safety and tolerability, as well as the objective response rate for non-irradiated metastases. $10(40 \%)$ patients needed immunosuppressive therapy with prednisone to treat classic immune-mediated adverse events observed with the dual combination. Radiation pneumonitis limited by the radiation field (grade 2 toxicity) was observed in 2 patients and responded quickly to oral steroids. At the time of the initial analysis, partial responses were recorded in 14 out of 25 patients, which amounted to $56 \%$. After a few months, the objective response rate increased to $68 \%$ (17 out of 25 patients). There was no progression in the irradiated lesions. H. Hammers et al. concluded that the approach with combination of radiation and immunotherapy in metastatic RCC has acceptable safety and promising antitumor activity, which requires further research. Ongoing phase II trial (CYTOSHRINK) randomizes untreated advanced RCC patients in a 2:1 fashion to ipilimumab/ nivolumab plus stereotactic body radiation therapy (30-40 Gy in 5 fractions) to the primary kidney mass between cycles 1 and 2 (experimental arm), versus ipilimumab/nivolumab alone (standard arm) [37]. Authors hypothesizes that stereotactic radiotherapy to the primary tumor will enhance the total efficacy of immunotherapy.

\section{FUTURE PROSPECTS OF NIVOLUMAB}

What are future prospects of nivolumab in kidney cancer therapy? First, these are combinations with targeted drugs. The results of the randomized phase 3 CheckMate 9ER trial, which evaluated the efficacy of the checkpoint inhibitor nivolumab in combination with the tyrosine kinase inhibitor cabozantinib as the first-line therapy for metastatic clear-cell RCC, were presented at ESMO 2020 [38]. 651 patients were stratified by the IMDC risk score, PD-L1 expression and region and randomized at 1:1 to receive nivolumab $240 \mathrm{mg}$ IV every 2 weeks plus cabozantinib $40 \mathrm{mg}$ orally once a day $(\mathrm{N}=323)$ or sunitinib $50 \mathrm{mg}$ orally on a $4 / 2$ schedule $(\mathrm{N}=328)$. Treatment was given until disease progression or unacceptable toxicity. The primary endpoint was progression-free survival as measured by a blinded independent centralized review. Secondary endpoints included overall survival, objective response rates, and safety.

With a median follow-up of 18.1 months, all three endpoints were met. The combination vs. sunitinib significantly increased progression-free survival $(\mathrm{HR}=0.51, P<0.0001$; median 16.6 vs. 8.3 months, respectively), overall survival $(\mathrm{HR}=0.60$; $P=0.0010$; medians not reached) and objective response rates $(55.7 \%$ vs. $27.1 \% ; P<0.0001) .8 \%$ and $4.6 \%$ of patients achieved complete response. The median duration of response was 20.2 months vs. 11.5 months. Grade $\geq 3$ treatment-related adverse events were reported in $60.6 \%$ of patients in the combination group and in $50.9 \%$ in the sunitinib group. Toxicity resulted in discontinuation of sunitinib in $8.8 \%$ of patients, nivolumab or cabozantinib in $15.3 \%$ of patients, the combination in $3.1 \%$ of patients, nivolumab alone in $5.6 \%$ of patients, and cabozantinib alone in $6.6 \%$ of patients. Thus, the combination of nivolumab and cabozantinib proved to be effective, which allows considering it as first-line therapy for metastatic clear cell RCC. On the other hand, higher toxicity grades, when compared with the results of the CheckMate 214 study, may somewhat limit its applicability, for example, in patients with comorbidities or at risk of cardiac toxicity. Longer follow-up will allow collecting more data.

Another direction of using nivolumab may be its combination with a fundamentally novel class of antibodies that inhibit immune checkpoints. For example, the fully human anti-LAG3 antibody relatlimab $(80 \mathrm{mg})$ plus nivolumab $(240 \mathrm{mg})$ are already being studied in clinical trials, including those in RCC patients. LAG3 is a protein that binds molecules of the major histocompatibility complex (MHC class II), thereby significantly suppressing the activity of the immune system. The triple blockade of PD-1, CTLA4 and LAG3 is also of scientific interest. Recently, a phase $1 / 2$ clinical trial (NCT03459222) was started to evaluate the safety and preliminary efficacy of a combination of relatlimab, nivolumab, and ipilimumab in two cohorts.

Third, attempts are made to move immunotherapy from the first-line treatment for metastatic RCC to adjuvant and neoadjuvant regimens. Many oncologists participate in a randomized phase 3 PROSPER trial (NCT03055013), in which patients with RCC stage $\mathrm{T} 2$ or higher and regional lymph node metastases are started on nivolumab before surgery and then continue it after surgery. The classic "adjuvant" 
phase 3 CheckMate 914 study evaluates the efficacy of nivolumab plus ipilimumab in patients at high risk of disease progression after surgery (NCT03138512).

And finally, could combination immunotherapy supersede surgery in patients with small kidney tumors in future? Indeed, $10 \%$ of patients experienced disappearance of all metastases with nivolumab plus ipilimumab in the CheckMate 214 study, which means there is a possibility of disappearance of small tumors in localized RCC. A pilot phase 2 trial (NCT04134182) evaluates complete response rates in T1aN0M0 kidney cancer patients receiving the combination and these results will be available in the near future [39].

In conclusion, the extended follow-up presented in different trials provides decisive evidence for the clinically relevant and long-term benefits of the nivolumab as monotherapy and in combinations in patients with metastatic RCC and continues to support checkpoint inhibitors as treatment option for this patient population. Novel combinations and studies will expand the value of immunotherapy.

\section{ACKNOWLEDGMENTS}

The author has no acknowledgments.

\section{FUNDING}

The author reports no funding.

\section{CONFLICT OF INTEREST}

The author declares no conflicts of interest.

\section{REFERENCES}

[1] Sayapina MS, Savyolov NA, Lyubimova NV, Timofeev Yu $\mathrm{S}$, Nosov DA. Outcome of metastatic renal cell carcinoma (mrcc) patients treated by anti-pd-1 therapy in expanded access program: clinical efficacy and potential biomarkers for nivolumab therapy. Malignant tumours. 2018;8(3):3138. (In Russ.).

[2] Porta C, Bonomi L, Lillaz B, et al. Renal cell carcinomainduced immunosuppression: an immunophenotypic study of lymphocyte subpopulations and circulating dendritic cells. Anticancer Res. 2007;27(1A):165-73.

[3] Tsimafeyeu I, Volkova M, Olshanskaia A, et al. Expression of Receptor Tyrosine Kinases on Peripheral Blood Mononuclear Cells and Tumor-Infiltrating Lymphocytes in Patients with Renal Cell Carcinoma and Healthy Donors. Oncology. 2020;98(4):252-8. doi: 10.1159/000505373. Epub 2020 Feb 13. PMID: 32053815.

[4] Ueda K, Suekane S, Kurose H, et al. Prognostic value of PD-1 and PD-L1 expression in patients with metastatic clear cell renal cell carcinoma. Urol Oncol. 2018;36(11):499.e9499.e16.

[5] Zhang X, Yin X, Zhang H, et al. Differential expressions of PD-1, PD-L1 and PD-L2 between primary and metastatic sites in renal cell carcinoma. BMC Cancer. 2019;19:360.

[6] Kahlmeyer A, Stöhr CG, Hartmann A, et al. Expression of PD-1 and CTLA-4 Are Negative Prognostic Markers in Renal Cell Carcinoma. J Clin Med. 2019;8(5):743.

[7] Meylan M, Beuselinck B, Dalban C, et al. Kidney ccRCC immune classification (KIC) enhances the predictive value of $\mathrm{T}$ effector (Teff) and angiogenesis (Angio) signatures in response to Nivolumab (N). ESMO Virtual Congress 2020. Abstract 700O.

[8] Demidov LV, Kharkevich GYu, Timofeev IV. Successes and failures in the use of cytokines in the drug therapy of some solid tumors. Practical Oncology, 4 (3-2003), 140.

[9] NCCN guidelines: Kidney Cancer, Version 3.2015. doi: 10.6004/jncen.2015.0022

[10] Tsimafeyeu I, Demidov L, Kharkevich G, et al. Granulocyte-Macrophage Colony-Stimulating Factor, Interferon Alpha andInterleukin-2 as Adjuvant Treatment for HighRisk Renal Cell Carcinoma. J Cancer Sci Ther. 2010;2:15759. doi:10.4172/1948-5956.1000042

[11] Xu JX, Maher VE, Zhang L, Tang S, Sridhara R, Ibrahim A, Kim G, Pazdur R. FDA Approval Summary: Nivolumab in Advanced Renal Cell Carcinoma After Anti-Angiogenic Therapy and Exploratory Predictive Biomarker Analysis. Oncologist. 2017;22(3):311-7. doi: 10.1634/theoncologist. 2016-0476.

[12] Motzer RJ, Rini BI, McDermott DF, et al. Nivolumab for Metastatic Renal Cell Carcinoma: Results of a Randomized Phase II Trial. J Clin Oncol. 2015;33(13):1430-7.

[13] Motzer RJ, Escudier B, McDermott DF, et al. Nivolumab versus Everolimus in Advanced Renal-Cell Carcinoma. N Engl J Med. 2015;373(19):1803-13.

[14] Motzer R, Tykodi S, Escudier B, et al. Final analysis of the CheckMate 025 trial comparing nivolumab (NIVO) versus everolimus (EVE) with $>5$ years of follow-up in patients with advanced renal cell carcinoma (aRCC). J Clin Oncol. 2020;38(suppl 6; abstr 617).

[15] Tsimafeyeu I, Zolotareva T, Varlamov S, et al. Five-year Survival of Patients With Metastatic Renal Cell Carcinoma in the Russian Federation: Results From the RENSUR5 Registry. Clin Genitourin Cancer. 2017;15(6):e1069-e1072.

[16] SEER database 2020. https://seer.cancer.gov/statfacts/html/ kidrp.html

[17] Cella D, Grünwald V, Nathan P, et al. Quality of life in patients with advanced renal cell carcinoma given nivolumab versus everolimus in CheckMate 025: a randomised, open-label, phase 3 trial. Lancet Oncol. 2016; 17(7):994-1003. doi: 10.1016/S1470-2045(16)30125-5. Epub 2016 Jun 6. Erratum in: Lancet Oncol. 2016 Jul;17 (7): 2270.

[18] Albiges L, Negrier S, Dalban C, et al. Safety and efficacy of nivolumab in metastatic renal cell carcinoma (mRCC): Final analysis from the NIVOREN GETUG AFU 26 study. J Clin Oncol 37, 2019 (no. 7_suppl (March 01, 2019) 542-542.). doi: 10.1200/JCO.2019.37.7_suppl.542

[19] Albiges L, Negrier S, Dalban C, et al. Safety and efficacy of nivolumab in metastatic renal cell carcinoma (mRCC): Results from the NIVOREN GETUG-AFU 26 study. J Clin Oncol. 2018;36:(suppl 6S; abstr 577).

[20] Flippot R, Dalban C, Laguerre B, et al. Safety and Efficacy of Nivolumab in Brain Metastases From Renal Cell Carcinoma: Results of the GETUG-AFU 26 NIVOREN 
Multicenter Phase II Study. J Clin Oncol. 2019;37(23): 2008-16. doi: 10.1200/JCO.18.02218

[21] Tsimafeyeu I, Gafanov R, Protsenko S, et al. Nivolumab in patients with metastatic renal cell carcinoma and chronic hepatitis $\mathrm{C}$ virus infection. Cancer Immunol Immunother. 2020;69(6):983-8. doi: 10.1007/s00262-020-02521-y

[22] Motzer RJ, Tannir NM, McDermott DF, et al. Nivolumab plus Ipilimumab versus Sunitinib in Advanced Renal-Cell Carcinoma. N Engl J Med. 2018;378(14):1277-90.

[23] Albiges L, Tannir NM, Burotto M, et al. Nivolumab plus ipilimumab versus sunitinib for first-line treatment of advanced renal cell carcinoma: extended 4-year follow-up of the phase III CheckMate 214 trial. ESMO Open. 2020;5:e001079. doi: 10.1136/esmoopen-2020-001079.

[24] Tsimafeyeu IV, Varlamov IS, Petkau VV, et al. Life expectancy in patients with metastatic renal cell carcinoma in the Russian Federation: results of the RENSUR3 multicenter registry study. Malignant tumours. 2019;9(2):45-52. (In Russ.) https://doi.org/10.18027/2224-5057-2019-9-245-52.

[25] Tannir NM, Signoretti S, Choueiri TK, et al. Efficacy and Safety of Nivolumab Plus Ipilimumab versus Sunitinib in First-line Treatment of Patients with Advanced Sarcomatoid Renal Cell Carcinoma. Clin Cancer Res. 2020 Sep 1.

[26] Tannir NM, McDermott DF, Escudier B, et al. Overall survival and independent review of response in CheckMate 214 with 42-month follow-up: First-line nivolumab+ipilimumab $(N+\mathrm{I})$ versus sunitinib $(\mathrm{S})$ in patients (pts) with advanced renal cell carcinoma (aRCC). J Clin Oncol. 2020; 38 (suppl 6; abstr 609).

[27] Nosov DA, Alekseev BYa, Volkova MI, Gladkov OA, Popov AM, Kharkevich GYu. Practical recommendations for drug treatment of renal cell carcinoma. Malignant Tumours: Practical Guidelines RUSSCO \# 3s2, 2020 (volume 10).31.

[28] NCCN Clinical Practice Guidelines in Oncology: Kidney Cancer, version 2.2020.

[29] McKay R, Xie W, McGregor B, et al. Optimized management of nivolumab (Nivo) and ipilimumab (Ipi) in advanced renal cell carcinoma (RCC): A response-based phase II study (OMNIVORE). J Clin Oncol. 2020;38:(suppl; abstr 5005).

[30] Atkins M, Jegede O, Haas N, et al. Phase II study of nivolumab and salvage nivolumab+ipilimumab in treatment-naïve patients (pts) with advanced renal cell carcinoma (RCC) (HCRN GU16-260). J Clin Oncol. 2020;38: (suppl; abstr 5006).
[31] Grimm M, Schmidinger M, Duran I, et al. Tailored ImmunoTherapy Approach with Nivolumab in advanced Renal Cell Carcinoma (TITAN-RCC). Annals of Oncology. 2019;30 (suppl_5): v851-v934. 10.1093/annonc/mdz394.

[32] Choueiri TK, Kluger HM, George S, et al. FRACTIONRCC: Innovative, high-throughput assessment of nivolumab+ipilimumab for treatment-refractory advanced renal cell carcinoma (aRCC). J Clin Oncol. 2020;38:(suppl; abstr 5007).

[33] Vano Y, Elaidi RT, Bennamoun M, et al. Results from the phase II biomarker driven trial with nivolumab $(\mathrm{N})$ and ipilimumab or VEGFR tyrosine kinase inhibitor (TKI) in naïve metastatic kidney cancer (m-ccRCC) patients (pts): The BIONIKK trial. Annals of Oncology, Volume 31, Supplement 4, 2020, Page S1157, Abstract LBA25.

[34] Dengina N, Tsimafeyeu I, Mitin T. Current Role of Radiotherapy for Renal-Cell Carcinoma: Review. Clin Genitourin Cancer. 2017;15(2):183-7. doi: 10.1016/j.clgc.2016.09. 004. Epub 2016 Sep 19.

[35] Dengina N, Mitin T, Gamayunov S, et al. Stereotactic body radiation therapy in combination with systemic therapy for metastatic renal cell carcinoma: a prospective multicentre study. ESMO Open. 2019;4(5):e000535. doi: 10.1136/ esmoopen-2019-000535.

[36] Hammers H, Vonmerveldt D, Ahn C, et al. Combination of dual immune checkpoint inhibition (ICI) with stereotactic radiation (SBRT) in metastatic renal cell carcinoma (mRCC) (RADVAX RCC). Journal of Clinical Oncology. 2020;38:no. 6_suppl (February 20, 2020):614-614.

[37] Lalani AA, Swaminath A, Pond GR, et al. Phase II trial of cytoreductive stereotactic hypofractionated radiotherapy with combination ipilimumab/nivolumab for metastatic kidney cancer (CYTOSHRINK). J Clin Oncol. 2020;38:6_ suppl, TPS761-TPS761.

[38] Choueiri T, Powles M, Burotto M, et al. 6960_PR Nivolumab+cabozantinib vs sunitinib in first-line treatment for advanced renal cell carcinoma: First results from the randomized phase III CheckMate 9ER trial. Annals of Oncology. 2020;31(suppl_4):S1142-S1215. 10.1016/ annonc/annonc325

[39] Tsimafeyeu I, Volkova M, Gafanov R, Nosov D, Bex A. A phase II pilot study of the efficacy and safety of nivolumab and ipilimumab in T1aNOM0 renal cell carcinoma patients ineligible for surgical treatment. Journal of Clinical Oncology. 2020;38:6_suppl, TPS769-TPS769. 